\title{
Hans-Bernd Massenmedien und Suizid: Brosius/ Praktische Konsequenzen aus Walther Ziegler dem Werther-Effekt
}

Der Werther-Effekt ist in der Medienwirkungsforschung ein viel beachtetes Phänomen. Seit der bahnbrechenden Studie des amerikanischen Soziologen Phillips (1974) wurde in zahlreichen Erhebungen versucht, Nachahmungstaten im Gefolge von realen und fiktiven Suiziden zu quantifizieren und zu evaluieren. Im folgenden Artikel werden die wichtigsten Stationen auf dem Weg der Erforschung des Werther-Effektes nachgezeichnet. Im Zentrum stehen dabei die praktischen Konsequenzen, die sich für eine verantwortungsvolle Berichterstattung über Suizide ergeben. Denn trotz vielfältiger und detaillierter Befunde zum Werther-Effekt hat es in Deutschland bisher keine Versuche zu einer praktischen Umsetzung der Forschungsergebnisse in einem suizidpräventiven Programm gegeben. Während in anderen Ländern bereits erste Richtlinien für die journalistische Berichterstattung ausgearbeitet und zum Teil implementiert wurden, sind solche Bemühungen in Deutschland erst in ihren Anfängen. Der Aufsatz extrahiert die konkreten Nachahmungsmuster bei Suiziden und die daraus resultierenden Konsequenzen für eine verantwortungsvolle journalistische Berichterstattung.

\section{Der Werther-Effekt: Hypothese oder Phänomen?}

Als Goethe Ende des 18. Jahrhunderts seinen Roman "Die Leiden des jungen Werther" veröffentlichte, löste der darin beschriebene Selbstmord der Hauptperson eine ganze Reihe von Suiziden in Europa aus. Während einige Forscher von einer "Suizid-Epidemie“ (Rothmann 1971) im Gefolge der Publikation sprechen, verweisen andere auf die rückwirkend nur unzureichende epidemiologische Erfassung dieses Phänomens (Steinberg 1999).

Durch Quellen belegt ist in jedem Fall eine zweistellige Zahl von Suiziden in verschiedenen europäischen Ländern, die in direkte Verbindung mit Goethes Buchpublikation gesetzt werden. Das Phänomen der Nachahmung des literarischen Vorbildes war bei diesen Fällen insofern evident, als sich die Suizidenten genau wie die tragische Romanfigur in blaue Jacke und gelbe Weste kleideten oder das Buch direkt beim Suizid bei sich führten, wie im Fall eines jungen Mannes namens Karstens, der sich bei aufgeschlagenem Buch erschoss, oder Christine von Lassberg, die sich mit dem Buch in der Tasche ertränkte (Steinberg 1999). 
Nach der Selbsterschießung des 18-Jährigen Karl von Hohenhausen im Jahre 1833 klagte dessen Mutter Goethe sogar nach dessen Tod als Schuldigen an: „Auch mein Sohn hatte mehrere Stellen im Werther angestrichen ... von euch wird Gott Rechenschaft fordern über die Anwendung eurer Talente" (Apell 1896: 117). Goethe selbst erkannte ebenfalls die fatale Medienwirkung seines Briefromans, war persönlich bei der Bergung der Leiche von Christine von Lassberg anwesend (Steiger 1982) und beteiligte sich voller Schuldgefühle (Wilkes 1989) an der Errichtung einer Gedenkstätte (Felsentor) zu Ehren der Toten. Er schrieb rückblickend: "Die Wirkung dieses Büchleins war groß, ja ungeheuer" und verglich sie mit einem "geringen Zündkraut", das eine "gewaltige Mine" zur Explosion brachte. Er schrieb wörtlich: „So verwirrten sich meine Freunde daran, indem sie glaubten, man müsse die Poesie in Wirklichkeit verwandeln (...) und sich allenfalls selbst erschießen: und was hier im Anfang unter Wenigen vorging, ereignete sich nachher im großen Publicum." (Goethe 1813: 630)

Der "Werther-Effekt" war geboren. Auch wenn die Nachahmungswirkung damals nicht mit quantitativen sozialwissenschaftlichen Methoden evaluiert werden konnte, handelte es sich keineswegs nur um eine Hypothese, sondern um ein aktenkundiges Phänomen, das schon zeitgenössisch als „Wertherfieber" bezeichnet wurde. Die Nachahmung des Selbstmordes des Romanhelden aufgrund einer Identifikation mit dem Modell wurde von Goethes Zeitgenossen als Tatsache angesehen. Dafuir spricht auch das Verbot des Buches in Leipzig, Kopenhagen und Mailand. Der Leipziger Stadtrat beispielsweise setzte am 30. Januar 1775 auf Empfehlung der theologischen Fakultät eine Strafe von 10 Talern auf den Verkauf und Handel des Buches mit der Begründung aus: „Es wird hier ein Buch verkauft, welches den Titel führt Leiden des jungen Werthers. Diese Schrift ist eine Empfehlung des Selbst Mordes; und es ist auch um des Willen gefährlich, weil es in einnehmender Schreib Art abgefaßt ist. ... Da die Schrift also tible Impressiones machen kann, welche, zumal bey schwachen Leuten, Weibs Personen, Eindrïcke machen kann, welche bey Gelegenheit aufwachen, und ihnen verführerisch werden können, so hat die theol. Fakultät für nöthig gefunden, zu sorgen, dass die Schrift unterdrickt werde: dazumal itzo die Exempel des Selbstmordes frequenter werden" (Wustmann1882). Das Verbot, das sogar untersagte, Werthertracht anzulegen, blieb bis 1825 in Kraft.

\section{Der Werther-Effekt als quantifizierbare Größe}

Auch wenn das Buch "Die Leiden des jungen Werther" speziell in der Sturm-und-Drang-Zeit eine Art Kultbuch unter den damaligen jungen 
Intellektuellen darstellte, blieb die Nachahmung von fiktiven und realen Suiziden kein zeitspezifisches Einzel-Phänomen der Goethe-Zeit, sondern ereignete sich exemplarisch in verschiedensten Variationen bis heute. Der amerikanische Soziologe David Phillips verwendete erstmals den Begriff „Werther-Effekt" als wissenschaftlichen Arbeitsbegriff zur Kennzeichnung von Nachahmungen medial vermittelter Suizide. Betrachtet man die historische Entwicklung, kommt solchen Nachahmungen gerade in modernen Medien-Gesellschaften sogar eine noch größere Bedeutung zu. $^{1}$

Zum ersten Mal gelang es Phillips 1974, die fatale Nachahmungswirkung der Berichterstattung über Suizide in einer systematischen und wissenschaftlichen Studie zu quantifizieren. Er stellte fest, dass nach Zeitungsberichten über Suizide prominenter Persönlichkeiten auch die Suizide in der Allgemeinbevölkerung statistisch meßbar ansteigen. Seine Methode war einfach: Er erstellte eine Liste mit 33 prominenten Personen, die in den Jahren 1947 bis 1967 Suizid begingen, über die dann auf der Titelseite der New York Times berichtet wurde und die zudem in "Facts on File“, einem Index der wichtigsten Weltnachrichten, verzeichnet waren. $\mathrm{Zu}$ diesen prominenten Selbstmördern zählten unter anderem auch der Klu Klux Klan-Führer Burros und die Schauspielerin Marilyn Monroe. Dann untersuchte Phillips die amtlichen Statistiken auf Schwankungen der Selbstmordhäufigkeit vor und nach Zeitungsberichten über den Selbstmord der aufgelisteten Prominenten, wobei er die Zahl der Suizide im Gefolge der Berichterstattung mit den Vorjahreszahlen und den Zahlen des darauffolgenden Jahres verglich. So nahm sich beispielsweise der Klu Klux Klan-Führer Burros am 1.November 1965 das Leben, nachdem bekannt wurde, dass er jüdischer Abstammung ist. In diesem Monat (November) wies die amerikanische Selbstmordstatistik 1.710 Suizide aus. Im selben Monat im Jahr zuvor gab es aber nur 1639 Fälle und auch ein Jahr später waren im entsprechenden Monat auch wieder nur 1665 Suizide zu verzeichnen. Der eigentlich zu erwartende Mittelwert von 1652 Suiziden unterschied sich also signifikant von der tatsächlichen Zahl im Gefolge der Zeitungsberichte über Burros' Tod. Insgesamt zeigte die Untersuchung von Phillips bei 26 von 33 Suiziden Prominenter einen signifikanten Folge-Anstieg der Suizide in der amerikanischen Bevölkerung, der um so deutlicher wurde, je größer die Promi-

1 Schon 24 Beginn des Jahrhunderts kamen Rost (1912) und andere Beobachter von Selbstmordepidemien zu der Vermutung, dass die Medien zu Nachahmungstaten anregen. Dies gilt nicht nur für Selbstmorde. Gabriel Tarde (1912) kam auch für Gewaltverbrechen zu dem Schluss, dass sich Verbrechen den Telegrafenleitungen folgend ausbreiten. 
nenz der Verstorbenen war. Am stärksten war dieser Effekt nach dem Suizid der Filmschauspielerin Marilyn Monroe.

Mit weiteren Befunden (u.a. wurde zusätzlich das Massenblatt New York Daily ausgewertet) konnte Phillips die These eines Nachahmungseffektes erhärten und spezifizieren: diejenigen Selbstmorde, über die in den Medien am intensivsten berichtet wurde, hatten den höchsten Anstieg zur Folge. Wurde nur ein Tag berichtet, betrug der durchschnittliche Anstieg 29 Suizide, bei zwei Tagen 35, bei drei Tagen 82 und bei vier Tagen (wie im Fall von Marilyn Monroe) 198 zusätzliche Suizide. Außerdem war der Anstieg am stärksten im geographischen Verbreitungsgebiet des untersuchten Mediums. Beiträge über Suizide, die beispielsweise nur auf den Titelseiten der New Yorker Zeitungen standen, nicht aber auf denen der Chicago Tribune hatten in New York einen größeren Effekt als in Chicago.

Auch wenn man jahreszeitliche Schwankungen, Einflüsse von Krieg und Wirtschaftszyklen herausrechnet, bleiben die von Phillips festgestellten signifikanten Effekte erhalten. Wassermann (1984) hat in einer Kontrollstudie die Datenbasis von Phillips erweitert und zusätzliche Einflüsse auf Schwankungen der Suizidalitätsrate kontrolliert, kam aber zu keinen wesentlich anderen Ergebnissen.

Phillips selbst ging dem Einwand nach, dass durch Zeitungsberichte über Suizide Personen ihre bereits getroffene Suizidentscheidung nur vorziehen würden. Diese Personen hätten sich über einen längeren Zeitraum hinweg ohnehin suizidiert. Insofern sei dem Werther-Effekt keine Verursachung von zusätzlichen Suiziden zuzuschreiben, sondern nur eine vorzeitige Auslösung. Phillips untersuchte deshalb, ob es nach einem signifikanten Anstieg der Suizidrate nach Medienberichten in den Folgemonaten zu einem entsprechend deutlichen Rückgang gekommen ist. Er kam zu dem Ergebnis, dass dies nicht der Fall ist und man deshalb davon ausgehen muss, dass durch Zeitungsberichte tatsächlich zusätzliche Suizide angeschoben werden, die sich ohne die Berichterstattung nicht ereignet hätten. Doch diese Schlussfolgerung lässt sich statistisch nicht eindeutig belegen, da unklar ist, welchen Zeitraum man für die Nachwirkungen $z \mathfrak{u}$ Grunde legen soll.

Bereits vier Jahre, bevor Phillips erstmals die Nachahmung von Suiziden nach Zeitungsberichten über Prominente evaluierte und den Begriff ,Werther-Effekt" verwendete, gelang es Motto (1970) aus der Beobachtung eines Zeitungsstreiks zumindest einen indirekten Hinweis auf den Nachahmungseffekt zu gewinnen. Er ging von der Annahme aus, dass während eines Medienstreiks keine Modell-Berichterstattung über Suizidhandlungen und somit auch kein Nachahmungseffekt möglich ist, was $\mathrm{zu}$ einem Absinken der Suizidrate führen müsste. Während eines 268- 
tägigen Zeitungsstreiks in Detroit fand er tatsächlich einen signifikanten Rückgang der Suizide, insbesondere bei jüngeren Frauen, und einen Wiederanstieg nach Beendigung des Streiks.

Ein methodisch überzeugender Nachweis des ,Werther-Effektes' gelang schließlich Schmidke \& Häfner (1986). Die Wissenschaftler werteten die Eisenbahnsuizide im Jahre 1981 nach der Ausstrahlung der sechsteiligen Fernsehsendung "Tod eines Schülers" im Zweiten Deutschen Fernsehen aus und registrierten eine hochsignifikante Zunahme der Suizide. $\mathrm{Zu}$ Beginn jeder der sechs dokumentarisch gehaltenen Sendungen sah man als Eröffnung immer dieselbe Szene am Bahndamm, an dem sich der Schüler vor einen herankommenden Zug warf. Bei den 15bis 19-jährigen Schülern, die dem Filmprotagonisten Claus Wagner am ähnlichsten waren, nahmen die Eisenbahnsuizide im Sendezeitraum um 175 Prozent $z u$, verglichen mit dem entsprechendem Zeitraum in den Jahren zuvor und danach. Als die sechsteilige Sendung eineinhalb Jahre später erneut ausgestrahlt wurde, wiederholte sich dieser Nachahmungseffekt. Die Suizidrate stieg ein zweites Mal deutlich an, in der Zielgruppe der 15- bis19-Jährigen diesmal um 115 Prozent.

Das ZDF ließ nach Bekanntwerden der Studie von Schmidtke und Häfner zwei Gegengutachten erstellen und veröffentlichte schließlich das Ergebnis in einer Pressemitteilung unter dem Titel: „Gutachter relativieren Mannheimer Studie - Kein ursächlicher Zusammenhang zwischen Fernsehserie und steigender Suizidrate Jugendlicher" (zitiert nach Zell, Schmidke, Häfner 1987: 132). Allerdings haben auch die vom ZDF beauftragten Gutachter die von Schmidke und Häfner vorgelegten Daten bestätigt. Jörns (1987) weist lediglich neben einigen hermeneutischen Einwänden gegen die Heranziehung von Banduras Lerntheorie darauf hin, dass im Einzelfall von Schmidke und Häfner nicht sichergestellt werden konnte, ob die Schüler, die sich im zeitlichen Gefolge der Ausstrahlung der Sendung suizidiert haben, die Sendung auch tatsächlich gesehen haben. Außerdem könnte das Ansteigen der Suizidrate auch als kulminierte Vorwegnahme später sowieso erfolgter Suizide interpretiert werden. Die Zahlen aber, welche die Deutsche Bundesbahn den Autoren gegeben hat, sind laut Jörns zuverlässig.

Auch Ringel (1986) stellte die wissenschaftlichen Ergebnisse der Studie keineswegs in Frage. Er weist lediglich darauf hin, dass das erklärte Ziel der Sendereihe ,Tod eines Schullers' die Sensibilisierung der Gesellschaft für Alarmzeichen bei Suizidgefährdeten gewesen sei und dass damit die notwendige öffentliche Diskussion des Themas angeregt wurde. Bezüglich des ungewollten Nachahmungseffektes aber bescheinigt Ringel den Autoren Schmidtke und Häfner eine "in Methode und Schlussfolgerung ausgezeichnete Arbeit" und hält eine "suggestive zur 
Nachahmung drängende Wirkung des Films" für wahrscheinlich (Ringel 1986: 60).

Der Nachahmungseffekt gilt wissenschaftlich inzwischen nicht mehr nur als Arbeitshypothese, sondern wird auch aufgrund einer ganzen Reihe von weiteren Befunden als gesichertes Phänomen (Welz 1992) angesehen. Sowohl Phillips als auch Häfner und Schmidtke wurde entgegnet, dass die Medienberichterstattung durch den Imitationseffekt lediglich diejenigen Suizide vorzeitig ausgelöst hat, die sich über einen längeren Zeitraum ohnehin ereignet hätten. Der "Werther-Effekt" stelle sich somit kurzfristig bei hochvulnerablen Gruppen ein, würde aber langfristig zu keinem zusätzlichen Ansteigen der Suizidrate führen (Diehl 1992). Diesem Haupteinwand wurde in Nachfolgeuntersuchungen nachgegangen. Wenn die Medienberichterstattung tatsächlich nur zu einem vorzeitigen Auslösen von Suiziden führen würde, müsste in der Folgezeit nach dem medial bedingten Anstieg der Suizidrate ein quantifizierbarer Rückgang zu verzeichnen sein. Schmidke und Häfner fanden für den Zeitraum von eineinhalb Jahren nach dem Anstieg kein entsprechendes überproportionales Absinken der Suizidrate (Schmidke \& Häfner 1986). Man muss also von der Möglichkeit ausgehen, dass durch Medienwirkungen Menschen zu Tode kommen, die sich normalerweise nicht das Leben nehmen würden.

In ihren Überblicksaufsätzen zum Forschungsstand kommen sowohl Goldney (1989), Welz (1992) als auch Brosius \& Esser (1995) zu dem eindeutigen Ergebnis: „Es besteht kein begründeter Zweifel mehr, dass die Medien zu Selbstmorden beitragen." Eine unreflektierte Berichterstattung ,wird zwangsläufig zu weiteren Selbstmorden führen“ (Goldney 1989: 33). Konsequenterweise fordern Phillips und Carstensen (1986: 688): „Pädagogen, Politiker und Journalisten mögen Wege in Betracht ziehen, wie man die Öffentlichkeit in weniger starkem Maße Berichten über Selbstmorde, sowohl insgesamt wie auch im Einzelfall aussetzt."

\section{Offizielle Richtlinien zur Suizidberichterstattung}

In der Tat kann man angesichts der Forschungsergebnisse zum WertherEffekt einen konkreten Handlungsbedarf ableiten. Offizielle Richtlinien $\mathrm{zu}$ einer suizidpräventiven Berichterstattung sind in den meisten europäischen Ländern nicht vorhanden oder zumindest verbesserungsfähig. So heißt es zum Beispiel in Punkt 8.4 des Deutschen Pressekodex zum Thema "Selbsttötung": „Die Berichterstattung über Selbsttötung gebietet Zuriickhaltung. Dies gilt insbesondere für die Nennung von $\mathrm{Na}$ men und die Schilderung näherer Begleitumstände. Eine Ausnahme ist 
beispielsweise dann zu rechtfertigen, wenn es sich um einen Vorfall der Zeitgeschichte von öffentlichem Interesse handelt" (Deutscher Presserat 1997: 17). Im Deutschen Pressekodex wird der „Werther-Effekt" nicht direkt angesprochen und das Schwergewicht mehr auf den Schutz der Privatsphäre gelegt. Fragwürdig erscheint angesichts der Forschungsergebnisse zur Nachahmung von Prominenten-Suiziden auch die großzügigere Behandlung von Suiziden im Zusammenhang mit Personen von öffentlichem Interesse.

Im Kapitel 7 der „Producer Guidelines“ der BBC (Producer-Guidelines 1996) hingegen wird seit 1996 bereits sehr konkret vor einem möglichen Nachahmungseffekt gewarnt: „Die Berichterstattung über Suizide könnte andere ermutigen. Berichte sollten Details der Suizidmethode vermeiden. Seien Sie besonders vorsichtig mit Details, wenn die Methode ungewöhnlich ist. ... Spezielle Sorgfalt sollte man bei Beurteilungen walten lassen, die den Eindruck erwecken, suizidales Verhalten und Suizidhandlungen $\mathrm{zu}$ entschuldigen oder zu glorifizieren."

Auch der Schweizer Presserat schreibt in einer eigenen Stellungnahme zur Berichterstattung über Suizid am 23. Dezember 1992: „Wegen der Gefahr der Nachahmung sind detaillierte Berichte über Suizide und Suizidversuche zu vermeiden. Dies gilt nicht nur für reale Fälle, sondern auch für fiktive in Kriminalfilmen, Beziehungsgeschichten, Milieufilmen usw. Die Frage der Medienwirkung ist beim Entscheid über die Publikation oder die Ausstrahlung eines Berichtes über einen Suizidfall mit zu berücksichtigen" (Schweizer Presserat 1992).

In vielen Ländern aber erfahren die wenigsten Journalisten in ihrer Ausbildung (Volontariat) und in der späteren Praxis von der Existenz des „Werther-Effektes“. Selbst die publizistischen Grundsätze der jeweiligen nationalen Presseräte sind den Redakteuren in der Regel nicht durchgängig bekannt. Es bedarf daher weitergehender Initiativen, als die bloße Festschreibung der Nachahmungsgefahr im jeweiligen nationalen Pressekodex, um eine suizidpräventive Berichterstattung zu erreichen. Um aber konkrete Empfehlungen zu implementieren, ist die genaue Kenntnis der konkreten Inhalte und Muster der Nachahmung wichtig. "Wie" wird imitiert und "was" wird imitiert? Auch wenn beide Fragen noch nicht erschöpfend beantwortet sind, lassen sich doch aus der Synopsis der Forschungsergebnisse der letzten 25 Jahre einige wesentliche Resultate gewinnen.

\section{Der Übertragungsweg}

Der "Werther-Effekt" als Wirkzusammenhang zwischen Modell-Suizid und Nachahmungstat wird mit der Imitationshypothese (Schmidtke 
1986), der Suggestionstheorie (Phillips 1974), dem Enthemmungseffekt (Bandura 1986) oder der Ansteckungshypothese (Bandura 1976) begründet. Trotz dieser terminologischen Vielfalt sind die faktischen Differenzierungen der Erklärungsmodelle minimal, insofern die Begriffe Suggestion und Enthemmung auf der einen Seite und Imitation und Ansteckung auf der anderen Seite nur verschiedene Perspektiven ein und des selben Sachverhaltes darstellen. Vom Modell aus, also vom ,SuizidVorbild' aus betrachtet, handelt es sich beim Werther-Effekt um ein ungewolltes ,Suggerieren“ und ,Beeindrucken'. Aus der Perspektive des ,Suizid-Nachahmers' handelt sich dagegen um ein ,Imitieren' bzw. ein ,sich anstecken' und ,identifizieren'. Je nach Sichtweise wird der Werther-Effekt mehr als passiver oder aktiver Vorgang konzipiert.

Als Erklärungsansatz wird in den meisten Studien auf die klassische Theorie des Modellernens von Bandura verwiesen. In Lernexperimenten konnte Bandura zeigen, dass der ganz normale menschliche Lernvorgang in der Kindheit, aber auch im späteren Erwachsenenleben, auf dem Nachvollzug von vorher gesehenen Verhaltensweisen, Ideen, Einstellungen und Urteilsweisen beruht. Die Ergebnisse dieses Lernvorgangs können der Erwerb völlig neuer Verhaltensweisen sein oder die Hemmung bzw. Enthemmung von bereits vorhandenen Verhaltensmustern. Der Lernvorgang verändert die Auftrittswahrscheinlichkeit von vorhandenen bzw. neuen Verhaltensweisen durch die am Modell beobachteten positiven bzw. negativen Konsequenzen eines solchen Verhaltens.

Auch der Werther-Effekt kann unter Heranziehung von Banduras Theorie als Ergebnis eines Lernvorgangs am Modell verstanden werden. Wird z.B. ein Suizid heroisiert, wird dieses Verhalten als akzeptabel und erfolgversprechend erlernt. Jede in Aussicht gestellte Anerkennung und sei es auch wie im Falle eines Suizids eine posthume Gratifikation kann die Ausführung einer suizidalen Tat fördern. Aus Banduras Theorie folgt auch, dass Modelle um so eher nachgeahmt werden, je ähnlicher der Nachahmende und das Modell sind. Auch dies lässt sich auf die Nachahmung von Suiziden übertragen. Von mindestens ebenso großer Bedeutung für das Verständnis der Wirkmechanismen des Werther-Effektes ist die Frage, was genau suggeriert bzw. „erlernt" und imitiert wird, also die Frage nach den Details des Werther-Effektes. Welche spezifischen Inhalte werden transportiert, imitiert und müssen folglich in einer suizidpräventiven Berichterstattung vermieden werden?

\section{Die konkreten Nachahmungsmuster bei Suiziden: der Ort}

Eine seit langem bekannte, gleichwohl erst in den 70er Jahren näher erforschte Tatsache ist die Attraktivität bestimmter Orte mit entspre- 
chenden natürlichen und baulichen Gegebenheiten. So wurden beispielsweise allein von 1948-1978 über 1.000 Suizide in Japan durch Sturz in den Mihara Yama Vulkan registriert. In den Vereinigten Staaten waren es 625 Suizide durch Sprung von der Golden Gate Bridge. In der diesbezüglichen Untersuchung von Seiden (1978) zeigte sich, dass nahezu die Hälfte der untersuchten Suizidenten ihren Wohnsitz außerhalb von San Francisco hatten. Auf ihrem Weg zur Golden Gate Bridge, wo sie sich zu Tode sprangen, mussten sie demnach zuvor die ebenso hohe Oakland Bridge überqueren.

In jeder Großstadt gibt es bestimmte, aus der Medienberichterstattung bekannte und daher präferierte Suizidorte. In München beispielsweise ist es die immer wieder fotografierte und in TV- und Zeitungsberichten abgebildete Großhesseloher Brücke, in Hamburg die Köhlbrandbrücke und ein bestimmtes Hochhaus in Eimsbüttel. Diese präferierten Lokalitäten sind den Suizidenten in den meisten Fällen vorher nicht aus eigener Anschauung bekannt, sondern wurden ihnen erst über die Berichterstattung der Medien als sichere, aufsehenerregende und bewährte Suizidorte vermittelt.

\section{Die konkreten Nachahmungsmuster bei Suiziden: die Methode}

Neben der Imitation des Suizidortes bietet natürlich auch die Berichterstattung über spezifische Suizidmethoden konkrete Anschauungsmuster für Nachahmungseffekte. So fand beispielsweise nach der plakativen $\mathrm{Me}$ dien-Berichterstattung über den Tod des in Deutschland sehr bekannten Politikers Uwe Barschel eine ganz neue, bis dahin fast unbekannte Suizidmethode eine multiplikative Verbreitung. Unter der Schlagzeile „Tod in der Badewanne" wurde 1987 über Wochen hinweg nicht nur das Foto des ertrunkenen Ministerpräsidenten in der Hotelbadewanne auf den Titelseiten der Zeitungen und Magazine vermarktet, sondern auch detailliert rekonstruiert, dass der Ministerpräsident in der mit Wasser gefüllten Wanne erst Schlafmittel und Alkohol zu sich genommen hat, infolgedessen ins Wasser geglitten und ertrunken ist, ohne dabei noch einmal zu Bewusstsein gekommen zu sein. Das Institut für Rechtsmedizin der Universität Hamburg verzeichnete daraufhin im Verlauf dieser exzessiven Berichterstattung in den letzten drei Monaten des Jahres 1987 allein im Stadtgebiet von Hamburg dreizehn weitere Todesfälle in der Badewanne, drei- bis viermal so viele wie in den Vergleichszeiträumen der Vorjahre (Truebner 1988). In Einzeluntersuchungen konnte in 6 Fällen über Aussagen von Angehörigen und Funde von Zeitungsausschnitten zum Thema "Tod in der Badewanne" am Suizidort ein direkter und konkreter 
Zusammenhang zu der Medienberichterstattung hergestellt werden. In Zeitschriften und TV-Sendungen hatte sich mehrfach auch die Gesellschaft für humanes Sterben zu Wort gemeldet und anlässlich des Suizids von Barschel detailliert die Vorgehensweise für die Durchführung des Badewannensuizides erörtert. Ein 22-jähriger ertränkte sich nach diesen Empfehlungen am Tag nach der Ausstrahlung, bei einer 77-jährigen fand sich ein herausgerissener Zeitungsausschnitt mit der genauen Anleitung für den Suizid in der Badewanne. In einem weiteren Fall hatte eine Großmutter zwei Tage vor ihrem Suizid die Nachricht vom Tode Barschels vernommen und ihrer Enkelin erklärt, dass sie sich auch „einen solch leichten Tod wünschen würde" (Truebner 1988: 417).

Wie sehr Presse- und TV-Berichte über konkrete Suizidmethoden zur Nachahmung anregen, zeigen auch die Erfahrungen nach dem Bekanntwerden der Wirkung des Pflanzenschutzmittels E605. Anlässlich des damals sehr beachteten Wormser Mordfalls Christa Lehmann wurde durch die Medien die starke Giftwirkung des Schädlingsbekämpfungsmittels einer breiten Öffentlichkeit bekannt gemacht. Im Gefolge dieser Berichterstattung kam es zu einer Selbstmordserie mit E605 (Pribilla 1954).

\section{Die konkreten Nachahmungsmuster bei Suiziden: der Modell- charakter}

Neben der Vermittlung von Suizidmethoden und Suizidorten durch die Medien wurden in verschiedenen Untersuchungen auch der Charakter, die soziale Identität und die Lebenssituation von Personen als potentielles Nachahmungsmuster erkannt. So zeigen die Untersuchungen von Phillips (1974) über Nachahmungstaten nach Prominentensuiziden, dass Personen, die zwar relativ bekannt, aber aufgrund ihres Charakters nicht besonders beliebt sind bzw. unemotionalisiert oder mit Gleichgiiltigkeit wahrgenommen werden, durch ihren Suizid weniger oder keine Nachfolgesuizide bewirken. Je beliebter die prominente Person und je größer die emotionale Nähe zur Leserschaft war, um so deutlicher führte ihr Suizid zu einem Ansteigen der Nachahmungstaten in der Bevölkerung, wie beispielsweise im Falle von Marilyn Monroe. Die Häufung der Suizidrate unmittelbar nach der Berichterstattung über den Tod der Schauspielerin wird u.a. auch auf die damals in den Medien beobachtbare Glorifizierung und Stilisierung ihres Suizides zurickgeführt. Es bleibt natürlich spekulativ, inwieweit das von ihr entworfene Medien-Bild des "Opfers" dabei eine katalysatorische Rolle gespielt hat und ein Werther-Effekt bei veränderter Berichterstattung hätte vermieden werden können. 
Neuere Untersuchungen nach dem Suizid von Kurt Cobain (Jobes et al. 1996) implizieren allerdings einen solchen negativen Effekt bei der Berichterstattung über Marilyn Monroe. Die Schauspielerin wurde tendenziell in den Medien als zu sensibel, emotional und zu gut für die berechnende und rauhe Filmwelt Hollywoods dargestellt, als eine verträumte schutzlose Künstlerin, die zum Opfer der Männer und der Filmindustrie wurde. Ihr Suizid durch Drogeneinnahme wurde stilisiert als verständlicher Ausweg, stiller Protest und endgültiger emotionaler Rückzug der Künstlerin in ihre Traumwelt. Auch wenn sich speziell die Wirkungen von solchen Idealisierungen und Heroisierungen nicht messen lassen, liegt der Verdacht nahe, dass durch die Heroisierung der Tat potentielle Nachahmer ermutigt wurden. Die in Aussicht gestellte posthume Anerkennung könnte bei vulnerablen Gruppen zu einer Enthemmung und Enttabuisierung der suizidalen Tat führen. ${ }^{2}$

Nach dem Suizid des Rockstars Kurt Cobain wollte man diesen „Monroe-Effekt" unter allen Umständen vermeiden. Als sich 7.000 Fans im Park von Seattle zu einer Lichterwache versammelten, befürchteten die Radiosender und die Stadtverwaltung einen Massensuizid. Daraufhin wurde der Leiter der psychiatrischen Klinik ans Mikrophon geholt, der die Trauernden aufforderte, sich die Hände zu geben, gemeinsam die nächsten Tage durchzustehen und sich gegenseitig zu trösten. Keiner sollte allein trauern. Er bot für alle eine Soforthilfe in der Klinik an und wies auf eine Beratungs-Hotline hin. Nur ein einziger junger Mann verließ die Versammlung und schoss sich in der selben Nacht in den Kopf. Auch in den darauf folgenden sieben Wochen blieb die Suizidrate nach offiziellen Angaben in King County, Washington, dem Heimatstaat von Kurt Cobain ohne den befürchteten signifikanten Anstieg. Zu Hilfe kam den Psychiatern auch eine Tonbandaufzeichnung von Courtney Love, der nicht minder bekannten Witwe von Cobain, die den Fans über

Der Suizid ist in westlichen Gesellschaften normalerweise ein starkes gesellschaftliches, religiöses und subjektiv moralisches Tabu. Bis in die 60iger Jahre waren beispielsweise "Selbstmordversuche' in England strafbar und bis 1983 bekamen ,Selbstmorder' in katholischen Ländern gemäß dem kanonischen Recht keine kirchliche Beerdigung auf geweihtem Boden. Die stärkste Hemmung ist das innere Tabu, Hand an sich zu legen, und damit eine schwere Verletzung des eigenen Lebens und des Lebens der Angehorigen und Hinterbliebenen $\mathrm{zu}$ verursachen. Diese einerseits kulturell tradierte und andererseits personliche Hemmschwelle vor autoagressivem Verhalten aus Furcht vor Strafe, Nichtanerkennung und Missachtung wird aber geschwächt bzw. aufgehoben, wenn durch prominente und idealisierte Personen demonstriert wird, dass der Suizid eine künstlerische Tat ist und somit eine für begabte und sensible Personen gangbare und mit Verständnis akzeptierte Handlungsalternative. 
Lautsprecher vorgespielt wurde. Courtney Love weinte zunächst und beklagte den Tod ihres Mannes, ging aber dann dazu über, ihn auch zu beschimpfen und zu verfluchen, weil er sich so habe gehen lassen. Diese Ambivalenz verhinderte einen Romantisierungseffekt und machte allen Anwesenden deutlich, dass Cobains Tod etwas Schreckliches ist. Zweitens machte das Bild seines brutal zerfetzten Gesichtes, das eine Zahnidentifikation erforderte, eine künstlerische Stilisierung wie bei Marilyn Monroe unmöglich, die sanft entschlafen war. Drittens gelang es den Psychiatern, bei den zahlreichen Anfragen der Medien eine differenzierte Berichterstattung zu erreichen. In eigens veranstalteten Pressekonferenzen und bei Einzelanfragen wurden die Journalisten über den WertherEffekt aufgeklärt, zur Verbreitung der Hilfe-Hotline aufgefordert und insbesondere detailliert über die persönliche Erkrankung des Patienten Kurt Cobain (gescheiterte Drogenentzugstherapie, unbehandelte Depressionen) informiert. Tatsächlich wurde in der Medienberichterstattung in TV, Radio und Printorganen bis auf wenige Ausnahmen die künstlerische Leistung des Musikers Cobain getrennt dargestellt von seinen gesundheitlichen Problemen und Drogenexzessen. "The general message was: ,great artist, great music ... stupid act, don't do it; here is where to call for help" (Jobes et al. 1996: 264).

Allerdings wurde der "Werther-Effekt" auch bei nicht prominenten Personen und bei Suiziden von fiktiven Personen festgesteilt. Auf breiter Basis untersuchte Stack (1990) die Suizidraten nach Titelgeschichten über Suizide von nicht-prominenten Personen im Zeitraum von 19681980. Dabei zeigte sich ebenfalls ein messbarer Anstieg im Gefolge der Berichterstattung. Allerdings fiel der Effekt im Durchschnitt etwa viermal geringer aus, als nach Berichten über Suizide von Prominenten. Dass auch fiktive Personen Nachahmungseffekte auslösen können, belegt die bereits erwähnte Untersuchung von Schmidke und Häfner (1986). Sie kamen zu dem Ergebnis, dass der Nachahmungseffekt nach der Ausstrahlung des Fernsehfilms "Tod eines Schülers" speziell in der Zielgruppe der 14- bis 19-jährigen männlichen Schüler im Vergleich zu anderen Geschlechts-, Alters- und Identitätsgruppen (Schülerinnen, Lehrlinge, 20- bis 40-Jährige) überproportional hoch war. Das heißt, die Nachahmung und Identifikation mit dem Suizid-Vorbild, in diesem Fall mit dem 19-jährigen Schüler Claus Wagner war am größten bei Personen, deren soziale und biologische Identität dem fiktiven Modell am nächsten kam.

Diese Erkenntnis wurde unterschätzt. Fatalerweise wollte die Fernsehserie „Tod eines Schülers“ junge Leute davor bewahren, sich selbst zu töten. Doch das pädagogische Ziel der Sendung, suizidinhibierend zu wirken, verkehrte sich in sein Gegenteil. Die Macher der Serie verzichteten zwar bewusst auf allzu reißerische Elemente und rekonstruierten in 
einem eher nüchtern gehaltenen Stil in jeder der sechs Folgen die Beziehungen des Schülers Claus Wagner zu seiner Umwelt: zu seinen Eltern, seinen Lehrern, seinen Mitschülern und seiner Freundin Inge. Aber gerade durch die Sorgfalt und lückenlose Schilderung seiner sozialen Identität und seiner persönlichen Probleme wurde seine Motivation klar und eindrucksvoll herausgearbeitet und für die größtenteils jugendlichen Fernsehzuschauer nachvollziehbar gemacht.

\section{Praktische Konsequenzen aus dem "Werther-Effekt"}

Fassen wir alle Einzelergebnisse der genannten Studien hinsichtlich der evaluierten Imitationswirkungen zusammen, ergibt sich ein facettenreiches Bild des Werther-Effektes. Als gefährliche Nachahmungs- und Identifikationsmöglichkeit zeigt sich die suizidale Tat als solche, die Person, die sie ausgeübt hat, die Motivation dieser Person, die Methode, der Ort und die Reaktionen, die der Suizid in der Nachwelt ausgelöst hat. Dabei ist die Identifikation mit dem Modell-Suizidenten bei der Bevölkerungsgruppe am stärksten, die der medial vermittelten ModellIdentität, also der Motivation, dem sozialem Umfeld, dem Alter, dem Aussehen und dem Geschlecht am nächsten kommt. In einem verantwortungsbewussten journalistischen Bericht müsste demnach im Sinne einer sorgfältigen Suizidprävention auf die Nennung folgender Informationen verzichtet werden.

- Angaben zur biologischen und sozialen Identität:

Detaillierte Angaben über Alter, Geschlecht und Aussehen (Fotos, Bilder) sollten ebenso vermieden werden, wie Angaben über soziale Beziehungen, emotionale Verfassung, Charakter und Leistungsfähigkeit (z.B. schulisches Versagen, Arbeitslosigkeit) der Suizidenten.

- Angaben zu Suizidmethode und Suizidort:

Vor allem Informationen über die Suizidmethode, die für potentielle Suizidenten instruktiv sein könnten, müssen vermieden werden. Der Suizidort darf auf keinen Fall mystifiziert werden (Die „Todesbrücke von München").

- Spekulationen über Ursachen und Bewertungen des Suizids:

Journalisten sollten sich jeglicher Wertung des Suizids enthalten. Nach Suiziden tendieren Angehörige und Bekannte oft dazu den Verstorbenen zu überhöhen, was sich in einer entsprechenden Berichterstattung niederschlägt ("Er blieb sich selbst treu, wollte sich nie anpassen“). Dies führt häufig auch zu einer Heroisierung („Sie lebte als Künstlerin seit jeher exzessiver und leidenschaftlicher als andere Menschen"). Simplifizierende Erklärungen ("In dieser Situati- 
on hatte er keine andere Chance mehr" oder "Sie war zu sensibel für diese Welt") sind ebenso zu vermeiden, wie Bewertungen des Suizids als unverständlich ("Er hatte doch alles, was es im Leben gibt") oder mitleiderregend ("Warum nur hat niemand gemerkt wie es in seinem Inneren wirklich aussah?"). Auch romantische Überhöhungen sind zu vermeiden. (,Sie wollten auf ewig vereint sein. Ihre Liebe war stärker als der Tod").

All diese Informationen und Bewertungen müssten im Sinne einer optimalen Suizidprävention vermieden werden, um den Werther-Effekt konsequent auszuschließen. Der Imperativ aus medizinischer und kommunikationswissenschaftlicher Sicht an Journalisten lautet demnach: „Vermeide jede Bewertung von Suiziden als heroisch, romantisch oder tragisch, um möglichen Nachahmern keine postmortalen Gratifikationen in Form von Anerkennung, Verehrung oder Mitleid in Aussicht zu stellen. Nenne weder den Namen des Suizidenten noch sein Alter und sein Geschlecht, um eine zielgruppenbezogene Identifizierung auszuschließen. Vermeide es ferner, die Suizidmethode und besonders - bei spektakulären Fällen - den Ort des Suizides zu erwähnen, um die konkrete Imitation unmöglich zu machen. Gebe aber vor allem keine Informationen über die Motivation, die äußeren und inneren Ursachen des Suizids und vermeide so jede Identifikationsmöglichkeit und Motivationsbrücke mit den konkreten Lebensumständen und Problemen des Suizidenten. Fazit: „Beschreibe den Suizidenten, die Methode, den Ort, die Lebensverhältnisse und die Gründe so abstrakt, dass sie kein Anschauungsmaterial mehr enthalten, das einer möglichen Identifikation und Enthemmung Vorschub leisten könnte!"

\section{Das Paradoxon der unanschaulichen Information}

Dieser, aus medizinischer Sicht konsequente und an dieser Stelle bewusst radikalisierte Imperativ stellt in seiner Reinform für Journalisten eine schier unlösbare Aufgabe dar. Selbst wenn man versucht, die Anforderungen an eine suizidpräventive Berichterstattung weniger radikal zu formulieren, bleiben unter dem Aspekt der möglichen Wirkung in jedem Fall drei Grundregeln zur Vermeidung des Werther-Effektes bestehen: (1) möglichst abstrakt und ohne Details, Abbildungen und Fotos zu berichten, (2) nicht über Hintergründe, Auslöser und Motive der Tat zu spekulieren und (3) stilistisch nüchtern zu bleiben, um Mitleid bzw. Betroffenheit zu vermeiden. Aber genau diese Empfehlungen widersprechen diametral den Regeln und Techniken, die der Journalist in seiner Ausbildung vermittelt bekommt (vgl. z.B. Pürer 1996): (1) unabhängig von 
Interessengruppen wahrheitsgetreu recherchieren und berichten und dabei die W-Fragen ("Wer hat was, wo, wann, wie, warum") zu beantworten, (2) die Öffentlichkeit über bisher unbekannten Hintergründe und Motive der Tat aufzuklären und (3) anschaulich und verständlich $z \mathfrak{u}$ berichten und Spannung und Betroffenheit zu erzeugen.

Journalisten kommen also hinsichtlich der Suizidberichterstattung in die paradoxe Situation im Interesse der Suizidprävention uninformativ, uninvestigativ und unattraktiv berichten zu müssen, also den Nachrichtenwert möglichst gering zu halten. Da im Konkurrenzkampf der Medienprodukte untereinander solche Artikel gerade deshalb keine oder geringe Publikationschancen haben, stellt sich die Frage, ob nicht aus journalistischer und medizinischer Sicht a priori gänzlich auf die Suizidberichterstattung bei konkreten Fällen verzichtet werden sollte. In Frankreich gibt es tatsächlich ein Pressegesetz aus dem Jahre 1955, das untersagt, den Suizid von Minderjährigen publik zu machen, sei es durch Bücher, Presse, Rundfunk, Kino oder in anderer Form. Diese "tragischen Ereignisse" (Jugend- und Kindersuizid), so heißt es in der Begründung, sollen durch das Verbot "vor jeder Publizität bewahrt" werden, die weitere Verzweiflungstaten veranlassen könnte (Guillon 1982). Aber dieses Pressegesetz geht auf den Werther-Effekt nur in bezug auf eine spezielle Altersgruppe ein.

In Punkt 1 der Stellungnahme des Schweizer Presserates zur Suizidberichterstattung heißt es zu diesem Problem unmissverständlich: „Suizide und Suizidversuche sind eine soziale Realität. Sie können für die Massenmedien grundsätzlich kein Tabu sein" (Schweizer Presserat Nr. 8/1992). Auch im Pressekodex des deutschen Presserates und den Produzenten-Guidelines der BBC wird die grundsätzliche Notwendigkeit der Suizidberichterstattung nicht in Frage gestellt. Es wird und soll also weiter über konkrete Suizide und Suizidversuche berichtet werden. Ein restriktives Total-Verbot ist aufgrund der Pressefreiheit und des Informationsrechtes der Öffentlichkeit weder durchsetzbar noch wünschenswert. So kann z.B. bei gehäuften Suiziden in Gefängnissen, psychiatrischen Kliniken, Schulen und anderen öffentlichen Anstalten eine Berichterstattung sogar hilfreich sein, eventuelle Missstände aufzuzeigen. Suizidberichterstattung muss also trotz der damit verbundenen Risiken möglich sein. Wie aber soll der Journalist mit dem Paradoxon der unanschaulichen Information umgehen? 


\section{Suizidberichterstattung zwischen Gesinnungs- und Verantwortungsethik}

Die Suizidberichterstattung bewegt sich auf einem schwierigen Feld. Der Journalist muss im Sinne einer suizidpräventiven Berichterstattung auf die detaillierte Wiedergabe des Sachverhalts verzichten. Andererseits ist er von seinem Berufsethos her gehalten, umfassend zu informieren, so dass ein bewusster Verzicht kaum möglich erscheint. Die verantwortlichen Chefredakteure und Journalisten vor Ort berufen sich insbesondere bei der Publikation von spektakulären Suiziden von Prominenten und Jugendlichen gerne auf das Interesse der Öffentlichkeit, dem sie nachzugehen verpflichtet sind und argumentieren gesinnungsethisch: „Die ganze Wahrheit muss berichtet werden."

Das Bekenntnis zur Wahrhaftigkeit als Grundwert der journalistischen Arbeit darf aber nicht darüber hinwegtäuschen, dass der Journalist jenseits dieser Gesinnung auch für die Folgen seiner Berichterstattung Verantwortung trägt. Erstmals hat Max Weber (1958) zu Beginn des zwanzigsten Jahrhunderts die grundlegende Unterscheidung zwischen Gesinnungs- und Verantwortungsethik vorgenommen und gleichzeitig auf deren notwendiges Zusammenspiel hingewiesen. Gesinnungsethisches Handeln erfolgt allein im Dienste der Überzeugungen von Werten ohne Rücksicht auf die voraussehbaren, vor allem negativen Folgen. Verantwortungsethisches Handeln erfolgt dagegen im Hinblick auf die aus dem Handeln resultierenden, beabsichtigten, aber auch unbeabsichtigten Folgen - macht also die Bewertungen der Konsequenzen zum Maßstab seiner Handlungen. Max Weber traf diese Unterscheidung in Bezug auf die politische Handlungsorientierung und wies darauf hin, dass eine Verabsolutierung der einen oder anderen Ausrichtung gefährlich sei. Der Politiker dürfe weder ausschließlich und rücksichtslos seiner Sittlichkeit und persönlichen Überzeugung folgen noch solle er sein Handeln prinzipiell nur nach den $\mathrm{zu}$ erwartenden Folgen seines Handelns ausrichten. Er müsse seine Überzeugungen mit ,Leidenschaft vertreten, gleichzeitig aber mit ,Augenmaß' und ,Verantwortungsbewusstsein' die Verhältnismäßigkeit der Mittel bedenken (Weber 1958).

Auch Journalisten geraten bei der Ausübung ihres Berufes häufig in das Spannungsfeld zwischen Gesinnungs- und Verantwortungsethik (vgl. Pürer 1992: 313): „Journalistisches Handeln vollzieht sich sehr oft auf der Basis weltanschaulicher Haltungen, muss andererseits aber - vor allem bei unbeabsichtigten negativen Folgen - vom einzelnen Journalisten verantwortet werden." Speziell bei der Suizidberichterstattung stehen Journalisten am Scheideweg zwischen Informationspflicht und medizinisch geforderter Zurückhaltung. Bei der Implementierung von 
suizidpräventiven Empfehlungen für die Berichterstattung kann es daher hilfreich sein, auf das von Max Weber skizzierte Zusammenspiel von Gesinnungs- und Verantwortungsethik hinzuweisen. Journalisten hätten demzufolge von Fall zu Fall eine Folgenabschätzung vorzunehmen und dabei die Risiken, Nachahmungstaten zu provozieren, gegenüber der Wahrheits- und Informationspflicht abzuwägen.

\section{Die Marktsituation: Suizidalität als Verkaufsfaktor}

Das Faktum der Marktorientierung der Medien, der zunehmende Konkurrenzdruck im Kampf um Auflagen und Zuschauer sowie der Zwang zur Aktualität und Exklusivität stellt für die Etablierung suizidpräventiver Empfehlungen eine weitere nicht zu unterschätzende Hürde dar. Dass die Berichterstattung sich nicht nur von Sorgfalts- und Informationspflicht leiten lässt, sondern immer auch von Marktimperativen, wird besonders in der zunehmend krisenhaften Entwicklung des Journalismus seit den 80er Jahren sichtbar. Das als Grusel-Story vermarktete Geiseldrama von Gladbeck mit Täterinterviews während der Geiselnahme ist nur ein Beispiel dafür. Hier wurde die polizeiliche Arbeit und damit das Leben der Geiseln massiv gefährdet, um dem Marktimperativ der sensationellen und hautnahen Berichterstattung gerecht $\mathrm{zu}$ werden. Es entsteht der Eindruck, dass einzelne Journalisten „mehr an ihre Karriere als an ihre Opfer denken, mehr an ihr Medium als an das Anliegen ihrer Branche, mehr an die Auflage als an ihre Verantwortung" (Weischenberg 1988: 10). Im Falle der Suizid-Berichterstattung sind die Imperative des Marktes nicht weniger gering. Ein Selbstmord eines Prominenten, der besonders tragische Freitod eines jungen Liebespärchens oder auch ein spektakulärer Suizid an einem ausgefallenen Ort sind marktstrategisch gesehen für Print- und TV-Medien ebenfalls reizvolle Aufhänger für eine auflagensteigernde Positionierung des Themas. Spätestens seit der Titelstory "Tod in der Badewanne" über den Ministerpräsidenten Barschel ist das Problem bekannt. Aus den legitimen „Human Touch Stories" wurde in einigen Fällen „Katastrophenjournalismus“ (Pürer 1991: 88). Dabei besteht der Markt aus anbietenden Medien und rezipierenden Konsumenten. Das Interesse der Bürger ist speziell bei Suiziden von Prominenten vorhanden und wird von den Medien konsequent bedient.

Aber selbst wenn es gelingt, eine Zeitungsredaktion für die Einhaltung suizidpräventiver Regeln zu gewinnen, würde dies für alle anderen konkurrierenden Blätter einen Marktvorteil bedeuten, sofern diese sich nicht auch auf die gleichen Regeln verpflichten lassen. Es ist daher bei der Etablierung von suizidpräventiven Empfehlungen von größter Wichtig. 
keit, alle relevanten Medien gleichzeitig, gemeinsam und unter gegenseitiger Beobachtung für den Verzicht auf bestimmte Formen der Suizidberichterstattung zu gewinnen. Nur so kann der, Systemdruck des Wettbewerbs' ausgeschaltet werden. Hierbei können Berufsverbände und $\mathrm{Ge}$ werkschaften wichtige Hilfestellung geben. Marktimperative der Berichterstattung lassen sich zwar nicht aufheben, aber es besteht die Chance, die Medien dafür zu gewinnen, bestimmte Formen der Suizid-Berichterstattung kollektiv aus dem Wettbewerb $z u$ nehmen. So kann eine freiwillige Selbstverpflichtung auf konkrete Regeln der Suizidberichterstattung unter Umständen tatsächlich erreicht werden.

\section{Suizidpräventive Medieninterventionen}

Eine erste quantifizierbar erfolgreiche Intervention dieser Art wurde in der österreichischen Hauptstadt durchgeführt (Etzersdorfer et al. 1994, Etzersdorfer \& Sonneck 1998). So kam es wenige Jahre nach dem Bau der U-Bahn in Wien in den Jahren 1984-87 zu einem dramatischen Anstieg der Suizide und Suizidversuche auf den Gleisen der Untergrundbahn, über die in den Medien intensiv und plakativ berichtet wurde. Als der österreichische Verein für Suizidverhütung (ÖVKSK) mit den Journalisten und Redaktionen Kontakt aufnahm und Empfehlungen für eine zurückhaltendere und suizidpräventive Berichterstattung ausarbeitete, konnte im Jahr 1987 die Suizidrate um 60 Prozent gesenkt und auch in den 5 Folgejahren auf dem niedrigeren Niveau stabilisiert werden. Der Medienguide enthielt neben inhaltlichen Empfehlungen, keine Details zu nennen und nicht zu emotionalisieren, auch Hinweise, um die generelle Wirkung und Verbreitung der Suizid-Nachricht herabzusetzen. So wurde empfohlen, über Suizide auf keinen Fall mit Bild oder Foto zu berichten, nicht auf der Titelseite und möglichst kurz gefasst. Die Einhaltung wurde über einen längeren Zeitraum beobachtet und in Fällen von erheblicher Nichtbeachtung der Richtlinien mit den entsprechenden Redaktionen und Journalisten Kontakt aufgenommen. Durch diesen nachhaltigen Kontakt mit den Wiener Medien konnte die Berichterstattung bis auf wenige Ausnahmen auf einem im Sinne der Suizidprävention akzeptablen Niveau gehalten werden. Dieses Beispiel könnte Medieninterventionen auf regionaler, nationaler und internationaler Ebene initiieren, die bei der Mehrzahl der Journalisten ein breites Verständnis für eine verantwortliche Berichterstattung schaffen können. ${ }^{3}$

3 Erstmals wird im Jahre 2001 auch in Deutschland im Auftrag der Bundesregierung (BMBF-Projekt) eine suizidpräventive Medienintervention durchgeführt. Im Rahmen 


\section{Literatur}

Apell, J.W. (41896): Werther und seine Zeit, Schulzesche HofBuchhandlung und Hof-Buchdruckerei, Oldenburg

Bandura, A. (1986): Social Foundation of Thought and Action. A Social Cognitive Theory. Englewood Cliffs, NJ, Prentice Hall

Bandura, A. (1976): Lernen am Modell, Klett-Verlag, Stuttgart

BBC Producer Guidelines (1996): Imitative and Anti-Social Behaviour, in: BBC Producer Guidelines 1996, http://www.bbc.co.uk/info/editioral/prodgl/07.htm

Brosius, H.-B., Esser, F. (1995): Eskalation durch Berichterstattung, Westdeutscher Verlag, Opladen

Deutscher Presserat (1997): Publizistische Grundsätze (Pressecodex), Richtlinien für die publizistische Arbeit nach den Empfehlungen des Deutschen Presserats, Bonn

Diehl, L.W. (1992): Einfluss der Suggestion auf Suizid. Kritik zur Vermittlung von Selbstmordmotivation und -handlung durch fiktive Modelle. Suizidprofilaxe 19 (2), 115-121

Etzersdorfer, E., Sonneck, G., Nagel-Kuess, S. (1994): Imitative suicide on the Viennese subway. Soc-Sci-Med. 38 (3), 453-457

Etzersdorfer, E., Sonneck, G. (1998): Preventing Suicide by Influencing Mass-Media Reporting. The Viennese Experience 1980-1996. Archives-of-Suicide-Research 4, 67-74

Goethe, J. W. (1813): Dichtung und Wahrheit, II.Teil, 13. Buch, zit. nach Gräf H. G. (1902) Goethe über seine Dichtungen. Versuch einer Sammlung aller Äusserungen des Dichters über seine poetischen Werke, Band I,2., Rüttten \& Loening Verlag, Frankfurt, S. $630 \mathrm{ff}$.

Goldney, R. D. 1989): Suicide: The role of media. Aust. N. Z. J. Psychiatry 23, 30-34

eines groß angelegten Awarenessprogramms in Numberg soll generell die Wirkung und Effizienz von suizidpräventiven Maßnahmen in einer kontrollierten Studie über mehrere Jahre evaluiert werden. Zu diesen suizidpräventiven Maßnahmen gehört auch die Implementierung eines Medienguides. Derzeit wird bereits in einem interdisziplinären Projekt von Psychiatern, Kommunikationswissenschaftlern und Journalisten ein solcher Wegweiser für eine adaquate Berichterstattung im Sinne der Suizidprăvention erarbeitet. Dieses Pilotprojekt in Deutschland ist aber nur einer von vielen Anläufen, die jetzt unternommen werden müssen, um die Theorie in die Praxis münden zu lassen. Nach einem Vierteljahrhundert der Forschung sind die Voraus. setzungen geschaffen, dem „Werther-Effekt" mit geeigneten Instrumenten entgegenzutreten. In westlichen Demokratien ist dabei zu erwarten, dass dies aufgrund der hohen Gewichtung der Pressefreiheit weniger durch legislative Maßnahmen von Seiten der Politik oder durch administrativen Zwang erfolgen wird, als durch die freiwillige Kooperation aller verantwortungsvollen Journalisten und Medien. 
Guillon, C., Y le Bonice. (1982): Gebrauchsanleitung zum Selbstmord. Robinson Verlag, Frankfurt a. Main

Jobes, D. A., A.L. Berman, P. W. O'Carroll, S. Eastgard, S. (1996): The Kurt Cobain suicide crisis: perspectives from research, public health, and the news media. Suicide Life Threat. Behav. 26, 269-271

Jörns, K.-P. (1987): Suizid als Thema in den Massenmedien Überlegungen $z u$ einer alten und neuen Kontroverse. Suizidprofilaxe 2 (1997), 61-78

Motto, J. A. (1970): Newspaper influence on suicide. Arch Gen Psychiatry 23, 143-148

Phillips, D. P. (1974): The influence of suggestion on suicide: substantive and theoretical implications of the Werther effect. Am. Soc. Rev. 39, 340-354

Phillips, D. P., Carstensen L. L. (1986): Clustering of teenage suicides after television news stories about suicide. New England Journal of Medicine 317, 685-689

Pribilla, 0. (1954): Vergiftungen mit E605. Arch.Toxicol.15, 210-284

Pürer, H. (1991): Journalismus, Krisen und Medien-Ethik, Elemente einer Ethik der Massenmedien, S. 88. In: Stuiber, H. W., Pürer, H.: Journalismus - Anforderungen Berufsauffassungen Verantwortung, Erlangen, S. 87-105.

Pürer, H. (1992): Ethik in Journalismus und Massenkommunikation. Publizistik 37, 304-321

Pürer, H. (1996): Praktischer Journalismus in Zeitung, Radio und Fernsehen, Salzburg

Ringel, E. (1986): Stellungnahme zum Film "Tod eines Schülers“. Suizidprofilaxe 2, (1997), 57-61

Rothmann, K. (1971): Erläuterungen und Dokumente zu Johann Wolfgang Goethe: Die Leiden des jungen Werther. Reclam-Verlag, Stuttgart, S. 193

Schmidtke, A., Häfner H. (1986): Die Vermittlung von Selbstmordmotivation und Selbstmordhandlung durch fiktive Modelle. Die Folgen der Fernsehserie "Tod eines Schülers". Der Nervenarzt 57 (9), 502-510

Schmidtke, A., Häfner H. (1988): The Werther effect after television films: new evidence for an old hypothesis. Psychological Medicin 18 (3), 665-676

Schweizer Presserat (1992): Stellungnahme des Presserates vom 23. Dezember 1992 zur Berichterstattung über Suizide, Nr.8/92

Seiden, R. H. (1978): Where are they now? A follow-up study of suicide attempters from the Golden Gate Bridge. Suicide-Life-Threat-Behav. $8(4), 203-16$ 
Stack, S. (1990): Audience receptiveness, the media, and aged suicide, 1968-1980. Journal of Aging studies 4, 195-209

Steiger, R. (1982): Goethes Leben von Tag zu Tag. Bd.II, Artemis Verlag, Zürich

Steinberg H. (1999): Der Werther-Effekt. Historischer Ursprung und Hintergrund eines Phänomens. Psychiat.Prax.26, 37-42

Truebner, K. (1988): Noch einmal: Tod in der Badewanne. Nach Barschels Selbstmord eine Häufung ähnlicher Fälle. Kriminalistik 42 (8-9), 414-418

Wassermann, I. (1984): Imitation and suicide: A reexamination of the Werther effect. American Sociological Review 49, 427-436

Weber, M. (1958): Politik als Beruf. In: Gesammelte politische Schriften. Hrsg. von Johannes Winkelmann, Tübingen

Weischenberg, S. (1988): Distanzverlust. Zwischen Information und Sensation. In: Der Journalist, H. 10, S. 10

Welz, R. (1992): Medien und Suizid: Zum Stand der Forschung, Suizidprofilaxe 19, 7-16

Wilkes, J. (1998): Mitschuldig am Suizid? Bewältigung von Trauer und Schuld durch Johann Wolfgang von Goethe. PPmP Psychosom. Med. Psychol. 48, 139-141

Wustmann, G. (1882): Verbotene Bücher. Aus den Censurakten der Leipziger Bücherkommission. Über das Verbot des „Werther“, in: Die Grenzboten. Zeitschrift für Politik, Literatur u. Kunst 41, 280-283

Zell, R. A., Schmidtke, A., Haefner, H. (1987): Kann Fernsehen Selbsttötung auslösen? Streit um die ZDF-Serie "Tod eines Schülers". Bild der Wissenschaft 24 (4), 122-132 